\title{
A Huge Aortic Aneurysm in a boy with Marfan Syndrome
}

\author{
*Ghaderian Mehdi and Ghafri Ghafar \\ Department of pediatric cardiology, Esfahan University of Medical Sciences, Iran
}

Submission: October 08, 2016; Published: October 21, 2016

*Corresponding author: Ghaderian Mehdi, Assistant professor of pediatric cardiology, Department of pediatric cardiology, Emam Hosein Medical, Educational and Research Center, Esfahan University of Medical Sciences, Emam Khomeini St., P.O.Box 8195100000, Esfahan, Iran.

\begin{abstract}
One of the most serious complications in Marfan's syndrome is dilation of aortic root and dissection of aorta and regularly physical examination of these patients is necessary. Dilatation of the ascending aorta is a rare finding in children with this syndrome. We present a 9.5-year-old boy; $22 \mathrm{~kg}$ weight was referred to our center because of systolic murmur during the routine examination for pre-operation of inguinal hernia. Transthoracic echocardiography and CT angiography showed a huge ascending aortic aneurysm. The patient underwent composite graft replacement of the aortic root and ascending aorta.
\end{abstract}

Keywords: Marfan Syndrome; Aortic Aneurysm

Abbreviations: CT: Computed Tomography; MFS: Marfan Syndrome

\section{Introduction}

In patients with Marfan's syndrome one of the most serious complications is dilation of aortic root and dissection of aorta. In these patients regularly physical examination and estimation and comparison of aortic root to previous examination is necessary for prevention of this complication.

In childhood dilatation of the ascending aorta is rare finding. It is usually associated with predisposing disease such as Marfan's syndrome (MFS). MFS is a genetic connective tissue disorder that its incidence is about 2-3 per 10000 individuals [1]. This disorder is a multisystem disorder involving many systems such as the cardiovascular and skeletal systems [2]. Aortic aneurysm and dissection of aorta remain the most life- threatening manifestations of patients with MFS. This finding is mostly age dependent, prompting life-long monitoring by echocardiography or other imaging modalities such as computed tomography angiography. Some unequivocally patients never reach an aortic size that needs surgical approach. Normal aortic diameter varies with both age and body size. The two most important risk factors that determinants dissection of the aorta is the family history of dissection and maximal dimension of aorta. When greatest diameter of aorta reaches about $50 \mathrm{~mm}$, surgical repair is recommended for prevention of dissection. In childhood there are no definitive describe to guide the timing of surgery. Early surgery is often undertaken given the emergence of significant aortic regurgitation or a rapid rate of growth ( $>1$ $\mathrm{cm}$ in a year).

\section{Case Report}

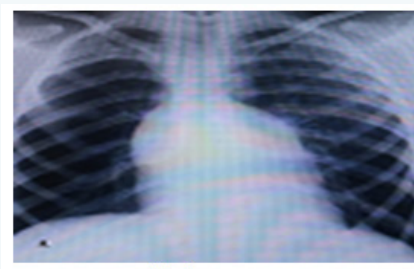

1(a)

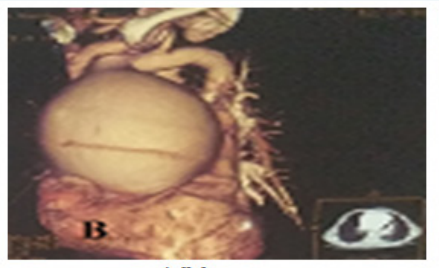

1 (b)

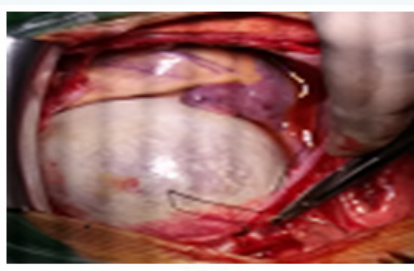

1 (c)

Figure 1: (a) Chest radiography showing a widened upper mediastinum with an enlarged upper segment of cardiac silhouette.

(b) 3-D reconstruction computed tomographic angiography of the chest showing a $7.5^{\star} 7.5^{\star} 11 \mathrm{~cm}$ ascending aortic aneurysm.

(c) Huge aneurysm during the surgery. 
A 9.5-year-old boy, $22 \mathrm{~kg}$ weight was referred to our center because of systolic murmur grade II/VI and mild chest deformity during the routine examination for pre-operation of inguinal hernia. He had some of physical features of the MFS including myopia $>3$ diopters, facial features, increased arm/height, hind foot deformity, deformity of the thoracic cage and inguinal hernia. Physical examination revealed chest deformity and a systolic murmur grade I-II/VI at the aortic area. Chest X-ray showed significant widened upper mediastinum (Figure 1).

Transthoracic echocardiography showed a huge ascending aortic aneurysm, that extending from sinotubular junction to aortic arch $(70 * 70 * 110 \mathrm{~mm})$ and mild aortic regurgitation.

CT angiography showed huge oval shape ascending aorta aneurysm $\left(75^{*} 75^{*} 116 \mathrm{~mm}\right)$ without dissection of aorta and normal aortic arch size. The patient underwent composite graft replacement of the aortic root and ascending aorta, without replacement of aortic valve or reimplantation of the coronary arteries into the graft.

\section{Conclusion}

In our case some of MFS feature including myopia $>3$ diopters, facial features, increased arm/height, hind foot deformity, chest deformity were seen. This case had dilation of ascending without dilation of sinuses of Valsalva. Huge aneurysm was reported in adult patients, but we could not find such huge aneurysm and Marfan syndrome in literature and articles in pediatric groups [3].

\section{Reference}

1. Daniel P Judge, Harry C Dietz (2005) Marfan's syndrome. Lancet 366(9501): 1965-1976.

2. Hiratzka LF, Bakris GL, Beckman JA, Bersin RM, Carr VF, et al. (2010) ACCF/AHNAATS/ACRI ASNSCNSCAIISIRISTS/SVM guidelines for the diagnosis and management of patients with Thoracic Aortic Disease: a report of the American College of Cardiology Foundation/ American Heart Association Task Force on Practice Guidelines, American Association for Thoracic Surgery, American College of Radiology, American Stroke Association, Society of Cardiovascular Anesthesiologists, Society for Cardiovascular Angiography and Interventions, Society of Interventional Radiology, Society of Thoracic Surgeons, and Society for Vascular Medicine. Circulation 121(13): e266-e369.

3. Dietz HC, Cutting GR, Pyeritz RE, Maslen CL, Sakai LY, et al. (1991) Marfan syndrome caused by a recurrent de novo missense mutation in the fibrillin gene. Nature 352(6333): 337-339.

4. Pinal-Fernandez, B Miranda-Barrio (2014) Giant aortic aneurysm in Marfan syndrome QJM 107(2): 155

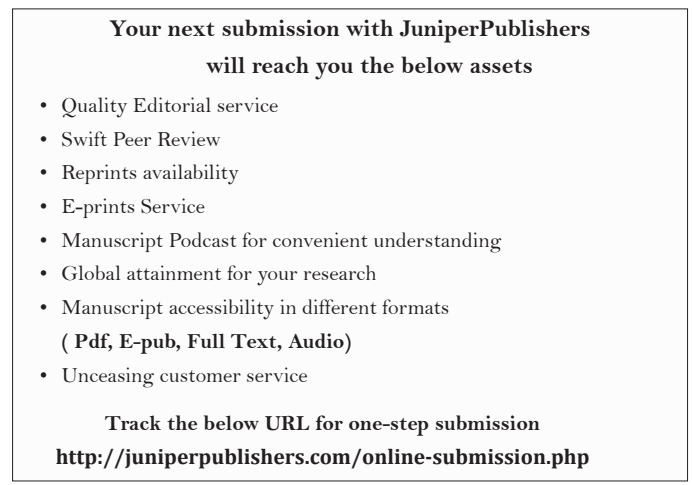

